HUB-EP-97/59

September 9, 1997

\title{
Hot electroweak matter near to the endpoint of the phase transition
}

\author{
M. Gürtler ${ }^{\mathrm{a}}$, E.-M. Ilgenfritz ${ }^{\mathrm{b}}$, A. Schiller ${ }^{\mathrm{a} *}$, and C. Strecha ${ }^{\mathrm{a}}$ \\ ${ }^{a}$ Institut für Theoretische Physik, Universität Leipzig, Augustusplatz 10-11, D-04109 Leipzig, Germany \\ ${ }^{\text {b} I n s t i t u t ~ f u ̈ r ~ P h y s i k, ~ H u m b o l d t-U n i v e r s i t a ̈ t ~ z u ~ B e r l i n, ~ I n v a l i d e n s t r . ~ 110, ~ D-10115 ~ B e r l i n, ~ G e r m a n y ~}$
}

The electroweak phase transition is investigated near to its endpoint in the framework of an effective threedimensional model. We measure the very weak interface tension with the tunneling correlation length method. First results for the mass spectrum and the corresponding wave functions in the symmetric phase are presented.

\section{The model}

The model under consideration is the $3 d$ $S U(2)$-Higgs model with the lattice action

$$
\begin{aligned}
S= & \beta_{G} \sum_{p}\left(1-\frac{1}{2} \operatorname{tr} U_{p}\right) \\
& -\beta_{H} \sum_{x, \mu} \frac{1}{2} \operatorname{tr}\left(\Phi_{x}^{+} U_{x, \mu} \Phi_{x+\mu}\right) \\
& +\sum_{x}\left(\rho_{x}^{2}+\beta_{R}\left(\rho_{x}^{2}-1\right)^{2}\right),
\end{aligned}
$$

$\rho_{x}^{2}=(1 / 2) \operatorname{tr}\left(\Phi_{x}^{+} \Phi_{x}\right)$. Our conventions and update details can be found in [1]. Relations between the parameters of this effective model and the physical quantities Higgs mass and temperature are derived in [2].

\section{Interface tension}

The interface tension $\alpha$ characterises the strength of a first order phase transition. We apply the tunnelling correlation method [3] to determine its value for a Higgs mass of about $70 \mathrm{GeV}$ by studying the inverse correlation length $m_{\text {lat }}=m_{\text {gap }} a$ (given by the correlation function of the Higgs operator $\rho_{x}^{2}$ along $z$ ) in an elongated lattice $L^{2} \times L_{z}$ as function of the transverse area $a^{2} L^{2}$. The scaling of $m_{\text {gap }}$ at the critical point is predicted for a $\phi^{4}$-model as $m_{\text {gap }} a L \propto x \exp (-x)$ with $x=\alpha a^{2} L^{2} / T_{c}$ [3] by considering quadratic fluctuations around the kink solution in a semiclassical expansion.

\footnotetext{
*Talk presented by A. Schiller
}

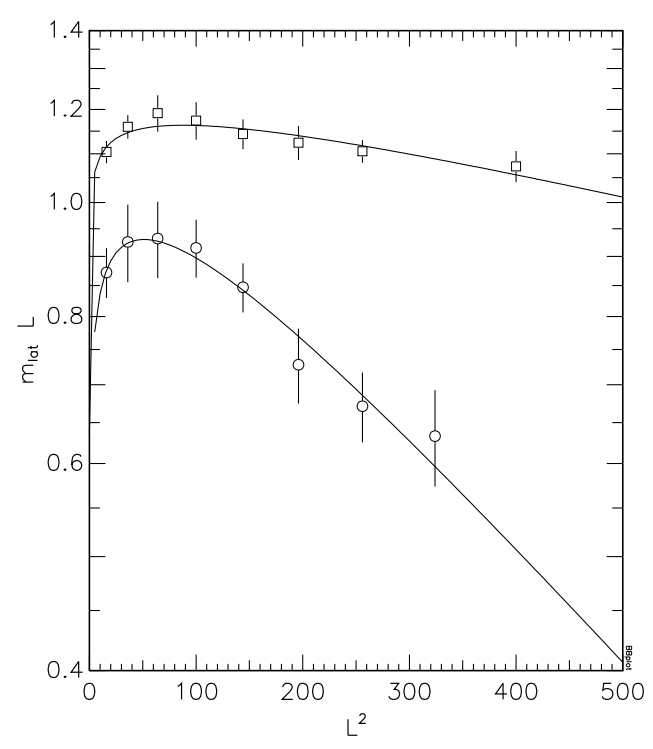

Figure 1. Fits for the inverse tunneling correlation lengths at $M_{H}^{*}=57 \mathrm{GeV}$ (circles) and $M_{H}^{*}=70 \mathrm{GeV}$ (squares)

The power of $L$ in the scaling law for $m_{\text {gap }}$ is eventually modified in higher orders. More general fits according to

$m_{\text {gap }} a L=c L^{\gamma} x \exp (-x)$

give the $3 d$ interface tension $\alpha_{3} / g_{3}^{4}=0.0049(18)$ (using $x=\left(\alpha_{3} / g_{3}^{4}\right) L^{2}\left(4 / \beta_{G}\right)^{2}$ for $3 d$ units) at $M_{H}^{*}=70 \mathrm{GeV}$.

As a check for the applicability of this method we have repeated the simulation for $M_{H}^{*}=$ $57 \mathrm{GeV}$ and obtained $\alpha_{3} / g_{3}^{4}=0.0224(56)$ in agreement with [4]. The measurements are shown in Fig. 目. 


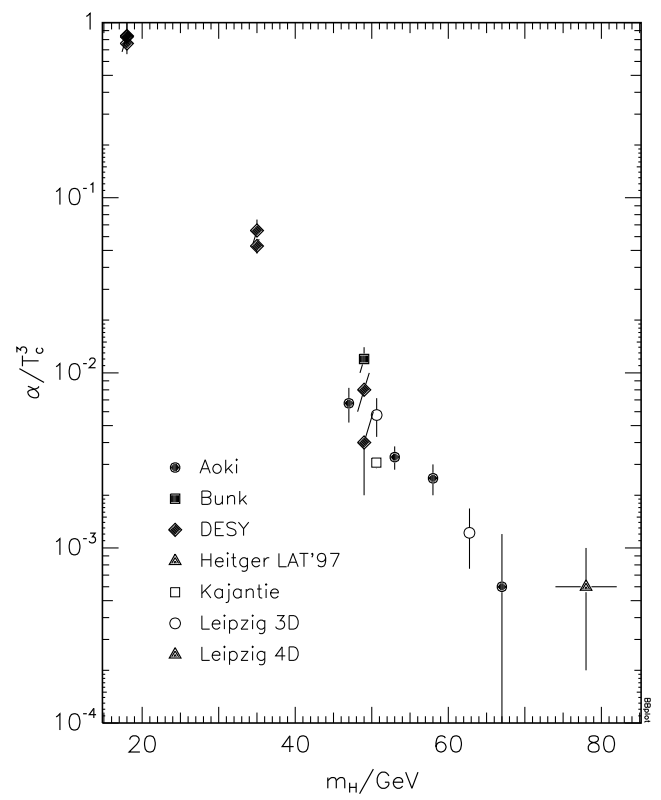

Figure 2. Interface tension for several Higgs masses; for references see $[5,6]$.

Fig. 2 collects published results for the interface tension (scaled by critical temperature) $\alpha / T_{c}^{3}$ of the $S U(2)$-Higgs model. In order to make this useful we had to recalculate the $3 d$ results to $4 d$ conventions (adjusting to a renormalised gauge coupling 0.58 characteristic for the $4 d$ lattice data). This comparison further supports the validity of dimensional reduction.

\section{Wavefunctions and excited states}

Knowledge of the mass spectrum of screening states in the symmetric phase is important to describe the high $T$ phase by non-perturbative analytic models. The wave functions may characterise the change between the phases. First studies have been reported recently [7].

To study ground state and excited states (and possibly wave functions) one has to use crosscorrelations between operators $\mathcal{O}_{i}$ from a complete set in a given $J^{P C}$ channel. In the transfer matrix formalism they describe the spectral decomposition $\left(\Psi_{i}^{(n)}=\left\langle\operatorname{vac}\left|\mathcal{O}_{\mathrm{i}}\right| \Psi^{(\mathrm{n})}\right\rangle,\left|\Psi^{(n)}\right\rangle\right.$ being the zero momentum energy eigenstates)

$C_{i j}(t)=\sum_{n=1}^{\infty} \Psi_{i}^{(n)} \Psi_{j}^{(n) *} e^{-m_{n} t}$ where $C(t)$ is the connected correlation matrix for distance $t$ in one of the spatial directions.

On the lattice one can use only a truncated set of operators $\mathcal{O}_{i}(i=1, \ldots, N)$ which is assumed to allow the extraction of the lowest lying states by considering the eigenvalue problem for $C_{i j}(t)$. Solving instead the generalised eigenvalue problem

$\sum_{j} C_{i j}(t) \Psi_{j}^{(n)}=\lambda^{(n)}\left(t, t_{0}\right) \sum_{j} C_{i j}\left(t_{0}\right) \Psi_{j}^{(n)}(4)$

errors related to this truncation can be minimised $\left(t>t_{0}, t_{0}=0,1\right)$ [8].

The wavefunction of state $n$ in the given operator basis is found (at fixed small distance $t>t_{0}$ ) to be

$\Psi_{i}^{(n)}(t)=\left\langle\operatorname{vac}\left|\mathcal{O}_{\mathrm{i}} \mathrm{e}^{-\mathrm{Ht}}\right| \Psi^{(\mathrm{n})}\right\rangle$.

The components characterise the contribution of the original lattice operators to the (ground or excited) state in the $J^{P C}$ channel. Using operators of different transverse extension (eventually only a subset of a used operator set with fixed quantum numbers) one can derive their relative contribution to an optimised wavefunction. The masses of these states are obtained fitting the diagonal elements $\mu^{(n)}(t)$

$\mu^{(n)}(t)=\sum_{i j} \Psi_{i}^{(n) *} C_{i j}(t) \Psi_{j}^{(n)}$

to cosh form.

Our base in the Higgs-channel $\left(0^{++}\right)$is built by the operators $\rho_{x}^{2}, S_{x, \mu}(l)$ as well as quadratic Wilson loops of size $l \times l$. In the $\mathrm{W}$-channel $\left(1^{--}\right)$ we use the operators $V_{x, \mu}^{b}(l)$ and in the $\left(2^{++}\right)$channel $S_{x, \mu}(l)-S_{x, \nu}(l)$. Here the following notation is used ( $l$ counts the string length in lattice units)

$$
\begin{gathered}
S_{x, \mu}(l)=\frac{1}{2} \operatorname{tr}\left(\Phi_{x}^{+} U_{x, \mu} \ldots U_{x+(l-1) \mu, \mu} \Phi_{x+l \mu}\right), \\
V_{x, \mu}^{b}(l)=\frac{1}{2} \operatorname{tr}\left(\tau^{b} \Phi_{x}^{+} U_{x, \mu} \ldots U_{x+(l-1) \mu, \mu} \Phi_{x+l \mu}\right) .
\end{gathered}
$$

As an example we show the squared wavefunction for ground state and first excitation in the $1^{--}$-channel in the high temperature phase near to the transition as function of the operator 
length $l$ for a $50^{3}$ lattice. The length is mapped to universal $g_{3}^{2}$ units in order to accommodate three $\beta_{G}$ values at the line of constant physics $m_{3}^{2} / g_{3}^{4} \approx 0.002$ (Figs. 3. 3. at $M_{H}^{*}=70 \mathrm{GeV}$.

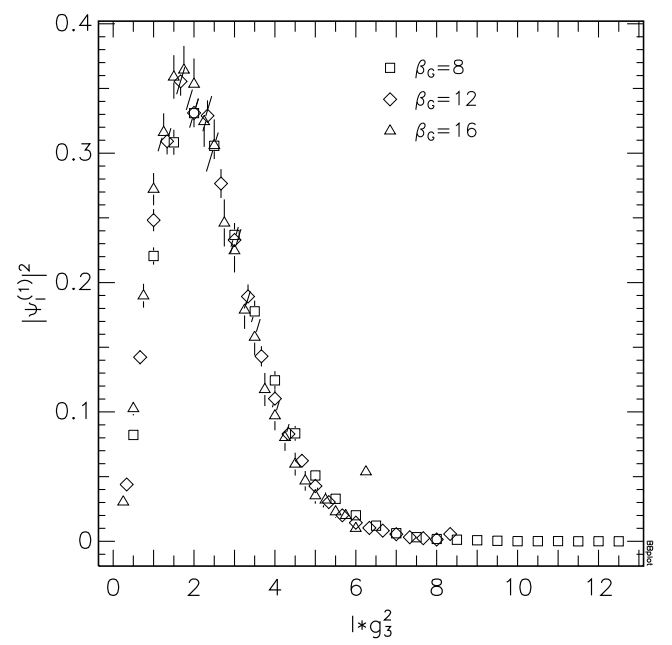

Figure 3. Squared wavefunction $\left|\Psi_{l}^{(1)}\right|^{2}$ for $1^{--}$ groundstate $n=1$ vs. physical length

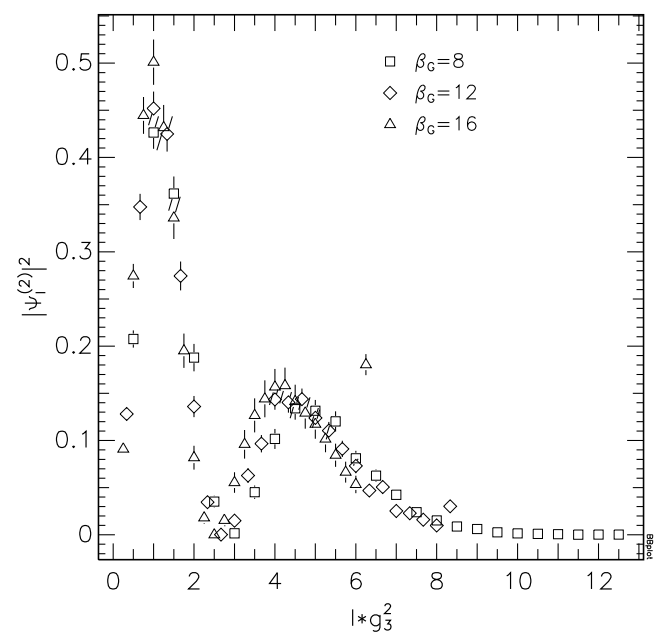

Figure 4. Same as Fig. 3 for first excited state $n=2$

A good scaling behaviour is observed. The last point $(l=25)$ seems to accumulate contributions from longer operators which do not fit to the lattice.

First results on the spectrum using these wavefunctions are presented in Fig. 5 showing to what

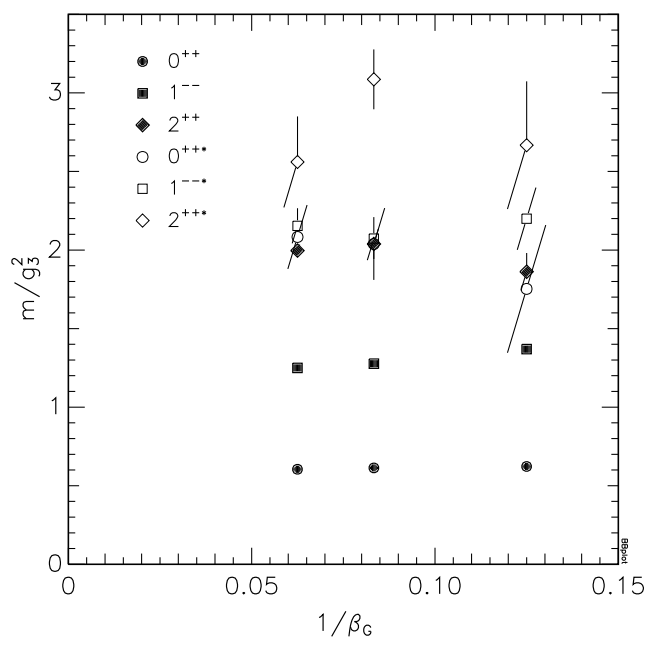

Figure 5. Masses of ground state and first excitation in physical units for different channels as function of $1 / \beta_{G} \propto a$.

extent different masses are already approaching the continuum limit. Aspects of different smearing procedures, mixing properties of excited states and results on different Higgs masses will be discussed in a future publication [9].

\section{REFERENCES}

1. M. Gürtler, E.M. Ilgenfritz, J. Kripfganz, H. Perlt, and A. Schiller, Nucl. Phys. B483 (1997) 383

2. K. Kajantie, M. Laine, K. Rummukainen, and M. Shaposhnikov, Nucl. Phys. B458 (1996) 90

3. G. Münster, Nucl. Phys. B340 (1990) 559

4. K. Kajantie, M. Laine, K. Rummukainen, and M. Shaposhnikov, Nucl. Phys. B466 (1996) 189

5. M. Gürtler, E.M. Ilgenfritz, and A. Schiller, hep-lat/9702020, to appear in Z.Phys.C

6. J. Heitger, these proceedings

7. O. Philipsen, M. Teper, and H. Wittig, Nucl. Phys. B469 (1996) 445

8. M. Lüscher and U. Wolff, Nucl. Phys. B339 (1990) 222

9. M. Gürtler, E.M. Ilgenfritz, A. Schiller, and C. Strecha, in preparation 